\title{
CARBONO NO SOLO, ACÚMULO E QUALIDADE DA SERAPILHEIRA EM SISTEMAS DE PRODUÇÃO FAMILIAR
}

\author{
Idelfonso Colares de Freitas $^{1 *}$, Felipe Corrêa Veloso dos Santos $^{2}$, Ronaldo de Oliveira Custódio Filho ${ }^{2}$, \\ Vladia Correchel ${ }^{2}$

\footnotetext{
1"Instituto Federal de Ciência e Tecnologia do Tocantins, Campus Araguatins, Araguatins, Tocantins, Brasil - idelfonsocolares@uol.com.br ${ }^{2}$ Universidade Federal de Goiás, Programa de Pós-Graduação em Agronomia (Solo e Água), Goiânia, Goiás, Brasil felipecvsantos@hotmail.com; rocf1983@gmail.com; vladiacorrechel@hotmail.com
}

Recebido para publicação: 09/07/2015 - Aceito para publicação: 12/10/2015

\begin{abstract}
Resumo
Na Amazônia, a conversão das florestas em agroecossistemas tem se caracterizado pela redução nos estoques de carbono vegetal, o que não se alinh a com os princípios da sustentabilidade. O objetivo deste estudo foi avaliar os impactos do uso do solo pela agricultura familiar nos estoques de carbono do solo, na quantidade acumulada e na qualidade da serapilheira. As formas de uso avaliadas foram: SAF = sistema agroflorestal, $\mathrm{PA}=$ pastagem cultivada, $\mathrm{RT}=$ roça de toco e MA = mata nativa, todos sobre Neossolo Quartzarênico. Em cada sistema foram coletadas sete amostras de serapilheira e de solo nas profundidades de 0-5, 5-10, 10-20 e 20-40 cm nos meses de julho de 2010 e janeiro de 2011. Foram analisados os teores de macro e micronutrientes, relação $\mathrm{C} / \mathrm{N}$, lignina, polifenóis e carbono na serapilheira e no solo. Entre os sistemas antropizados, o SAF apresentou maior produção de serapilheira e acúmulo de nutrientes nos resíduos nos dois períodos avaliados e maior estoque de carbono no solo no período seco. No entanto, seus resíduos foram mais recalcitrantes que os tratamentos MA e PA.

Palavras-chave: Sistema agroflorestal; agricultura itinerante; agricultura familiar.
\end{abstract}

\section{Abstract}

Carbon in soil, accumulation and quality litter in family production systems. In the Amazon, the conversion of forests into agricultural ecosystems has been characterized by a reduction in carbon stocks in vegetation, which does not align with the principles of sustainability. The aim of this study was to evaluate the impacts of soil use by family farmers in soil carbon stocks, quantity and quality of litter accumulated. Forms of use were evaluated: $\mathrm{SAF}=$ agroforestry system, $\mathrm{PA}=$ pasture, $\mathrm{RT}=$ stump field and $\mathrm{MA}=$ preserved forest, all about Quartzipsamment. In each system were collected seven samples of litter and soil at depths of 0-5, 5-10, 10-20 and $20-40 \mathrm{~cm}$ in July 2010 and January 2011. The contents of macro and micronutrients, C/N ratio, lignin, polyphenols and carbon in the litter and soil were analyzed. Among the anthropogenic systems the SAF system presented higher litter production, accumulation of nutrients in the residues in these two periods of collection and increased carbon storage in the soil during the dry season. However, their residues were more recalcitrant than those of the MA and PA treatments.

Keywords: Agroforestry system; shifting agriculture; family farming.

\section{INTRODUÇÃO}

Na Amazônia, a conversão das florestas em agroecossistemas tem sido marcada pela redução dos estoques de carbono (C) na biomassa, o que implica sua transferência para a atmosfera. Segundo Matheus (2012), as plantações e pastos podem ter de 20 a 100 vezes menos carbono estocado por unidade de área que as florestas, e a estimativa de transferência de C para a atmosfera, decorrente dessa conversão, corresponde a 1,7 bilhões de toneladas por ano. No solo, a quantidade de $\mathrm{C}$ armazenada chega a quase $1.500 \mathrm{Gt}\left(1 \mathrm{Gt}=10^{9}\right.$ toneladas), enquanto a atmosfera contém, aproximadamente, $750 \mathrm{Gt}$, ou seja, metade do C do solo. Assim, pequena mudança no carbono do solo pode afetar significativamente seus estoques na atmosfera.

O grande desafio da produção agropecuária na Amazônia é desenvolver agroecossistemas que aliem produção e conservação ambiental, sobretudo para a agricultura familiar que, tradicionalmente, cultiva sobre as cinzas produzidas pela combustão da vegetação natural. Uma alternativa, supostamente sustentável, que vem sendo apresentada para essa região é o uso de sistemas agroflorestais (MUTUO et al., 2005). Esses sistemas de produção, por manterem uma cobertura vegetal semelhante às áreas sob floresta, permitem a manutenção da fertilidade do solo fundamentada no aporte e ciclagem dos resíduos orgânicos (SILVA et al., 2011). Isso, além de garantir a sustentabilidade da produção, especialmente nos solos de baixa fertilidade da Amazônia, pelos benefícios da

FLOREST A, Curitiba, PR, v. 46, n. 1, p. 31 - 38, jan. / mar. 2016.

Freit as, I. C. de et al.

ISSN eletrônico 1982-4688

DOI: $10.5380 /$ rf.v46il 42065 
matéria orgânica nas propriedades físicas, químicas e biológicas do solo, possibilita grande acúmulo de carbono nos compartimentos biomassa, serapilheira e solo (MUTUO et al., 2005; FROUFE et al., 2011).

$\mathrm{Na}$ literatura, a maioria dos trabalhos relativos à serapilheira estuda a produção e o acúmulo em ecossistemas preservados (FERREIRA et al., 2007; SANCHES et al., 2009) ou agroecossistemas silviagrícolas (VIEIRA et al., 2009). Entretanto, estudos comparativos entre ecossistemas antropizados e preservados são importantes para fornecer subsídios no desenho e manejo de agroecossistemas sustentáveis, pois, nos solos de baixa fertilidade do trópico úmido, a matéria orgânica responde pela maior parte da capacidade de troca de cátions no solo (SILVA et al., 2011), sendo aportada ao solo via resíduos da parte aérea das comunidades vegetais que integram o agroecossistema (SANCHES et al., 2009).

No entanto, fatores ambientais, como elevadas temperaturas e umidade, característicos da região, favorecem a mineralização da serapilheira e da matéria orgânica do solo (CALDEIRA et al., 2007). Do ponto de vis ta de nutrição das plantas, essa rápida mineralização é importante, porém, quando se trata da imobilização do carbono, como forma de mitigação biológica dos efeitos do gás carbônico na atmosfera, configura-se como uma característica negativa. Nesse contexto, a natureza bioquímica dos resíduos orgânicos formadores da serapilheira é decisiva para o tempo de residência do material sobre o solo (SANCHES et al., 2009).

Nesse sentido, Tian et al. (1995) desenvolveram um modelo matemático que permite predizer a qualidade dos resíduos vegetais, o qual integra a relação $\mathrm{C} / \mathrm{N}$, conteúdo de polifenóis e lignina do resíduo, correlacionando-se muito bem com estudos desenvolvidos em sacos plásticos (littler bags). Trata-se de uma metodologia alternativa para estudos em que não se adaptam os coletores metálicos ou de madeira com fundo telado e que tem sido utilizada em estudos comparativos relativos à qualidade dos resíduos de plantas e restos culturais (A GUIA R et al., 2010a; A GUIA R et al., 2010b).

Nesse sentido, este trabalho testou a hipótese de que os sistemas de manejo do solo pela agricultura familiar afetam, de forma dife renciada, o carbono do solo e a quantidade e qualidade da serapilheira estocada, os quais podem ser utilizados como parâmetros para avaliação de sua qualidade. Dessa forma, conduziu-se este estudo com o objetivo de comparar indicadores de qualidade do solo em diferentes sistemas de manejo utilizados pela agricu ltura familiar, com vistas à definição de sistemas de manejo sustentáveis.

\section{MATERIAL E MÉTODOS}

O estudo foi realizado no lote 52 do Assentamento Tobasa, no município de Esperantina (Tocantins), Brasil, nas coordenadas geográficas $5^{\circ} 22^{\prime} 02^{\prime \prime} \mathrm{S}$ e $48^{\circ} 35^{\prime} 57^{\prime} \mathrm{O}$, e altitude média local de $90 \mathrm{~m}$. A precipitação média local é de $1.500 \mathrm{~mm}^{-a n o}{ }^{-1}$ e a temperatura média anual é de $28,5^{\circ} \mathrm{C}$. O clima, classificado como $\mathrm{Aw}$ (Köppen), apresenta seis meses de período chuvoso (dezembro a maio) e seis meses de período seco (junho a novembro). O solo foi classificado co mo Neossolo Quartzarênico Órtico $\left(\mathrm{RQ}_{0}\right)$, conforme sistema brasileiro de classificação de solos (SiBCS).

Os sistemas de manejo do solo avaliados foram:

1. SAF: sistema agroflorestal implantado em 1989 em volta da sede, onde convivem, livremente, 65 galinhas do tipo caipira. Evoluiu de um quintal agroflorestal para uma agrofloresta, com mais de sessenta espécies cultivadas em consórcio, sendo o cupuaçu (Theobroma grandiflorum) a principal cultura econômica do sistema, com produção anual de polpa de $1.500 \mathrm{~kg}$, seguida pela do bacuri (Platonia insignis), com produção anual de polpa de $750 \mathrm{~kg}$. Ocupa uma área de três hectares, na qual são adicionados, anualmente, resíduos oriundos da atividade doméstica, como cascas e sementes de frutas, cascas de mandioca e feijão, palhas e sabugos de milho, entre outros, estimando-se uma adubação orgânica anual de $1,2 \mathrm{Mg} \cdot \mathrm{ha}^{-1}$. Encontra-se em estágio de sucessão ecológica de capoeira grossa, com biomas sa aérea de, aproximadamente, $70 \%$ da observada visualmente na mata nativa.

2. PA: pastagem cultivada imp lantada em 1989 com capim-marmelada (Brachiaria plantaginea), localizada ao lado do sistema agroflorestal. Ocupa uma área de 16 hectares, usada no pastejo de bovinos, equinos e ovinos, mantendo uma pressão de pastejo constante de 0,8 unidades animal (UA) por hectare $(1 \mathrm{UA}=450 \mathrm{~kg}$ de peso vivo). O proprietário comercializa anualmente cinco bezerros de oito meses de idade, descarta duas matrizes para o abate (150 kg de carcaça) e ordenha quinze litros de leite diariamente.

3. RT: roça de toco, caracterizada pela retirada da floresta nativa, que teve sua fitomassa seca queimada em 2009, sendo utilizada para cultivo de arroz (Oryza sativa), milho (Zea mays), feijão trepa-pau (Vigna unguiculata) e mandioca (Manihot esculenta) até maio de 2010. Ocupa uma área de 2,5 hectares, na qual são colhidos, em média, $1.200 \mathrm{~kg}$ de arroz, $900 \mathrm{~kg}$ de milho, $120 \mathrm{~kg}$ de feijão e a mandioca (bianual), encontrando-se em fase de crescimento. Todas as espécies são cultivadas na forma de consórcio.

4. MA: mata nativa do tipo floresta aberta, com expressivo estoque de biomassa aérea, usada como testemunha. Localiza-se ao lado da roça de toco. É usada como reserva e, eventualmente, é colhida lenha, 
madeira para o fabrico de casas para moradia e frutas nativas da região.

Todos os sistemas antropizados tiveram como preparo in icial do solo o corte e queima da vegetação natural, sem uso de mecanização nem de adubos solúveis ao longo dos anos. Em cada sistema de uso do solo, foi delimitada uma área de 30 x $50 \mathrm{~m}$, em cujo interior foram marcados, aleatoriamente, sete pontos de amostragem. No entorno de cada ponto foram realizadas quatro coletas de serapilheira, utilizando-se um quadrado metálico de $0,50 \mathrm{~m}$ de aresta, para compor uma amostra composta, totalizando sete amostras compostas por sistema de manejo em cada período de amostragem. Após a coleta, o material foi pesado e dele foi retirada uma alíquota de peso conhecido, acondicionada em saco de papel e colocada em estufa de ventilação forçada à temperatura de $58^{\circ} \mathrm{C}$ até peso constante, para estimativa do rendimento de massa seca. Posteriormente, parte do material seco foi triturada em moinho tipo Willy com peneira de 30 mesh, acondicionada em pote plástico e identificada para análises.

As análises de $\mathrm{C}, \mathrm{N}, \mathrm{P}, \mathrm{K}, \mathrm{Ca}, \mathrm{Mg}, \mathrm{S}, \mathrm{Cu}, \mathrm{Fe}, \mathrm{Mn}$ e $\mathrm{Zn}$ da serapilheira seguiram metodologia usada por Morais et al. (2012). A lignina foi feita em análise sequencial de fibra detergente neutra (FDN), fibra detergente ácida (FDA) e lignina, enquanto os fenóis totais foram quantificados como taninos condensados, conforme procedimentos usados por Aguiar et al. (2010a).

Em cada período de coleta e ponto de amostragem foi aberta u ma minitrincheira. Em suas paredes, em posição mediana na camada amostrada, foram coletadas amostras de terra, com auxílio de anéis volumétricos com dimensões de $5 \times 5 \mathrm{~cm}$, para determinação da densidade do solo, e, com um facão, uma porção de aproximadamente $0,2 \mathrm{~kg}$ de solo para análise do carbono. Es te foi analis ado por digestão úmida e a densidade do solo pela relação entre a massa do solo seco e o volume total. As amostras de solo foram coletadas nas camadas de 0-5, 5-10, 10-20 e 20-40 cm, em duas épocas do ano, período seco (julho/2010) e período chuvoso (janeiro/2011).

Foi usado o índice de qualidade de resíduos vegetais (IQRV), proposto por Tian et al. (1995), calculado por meio da fórmula IQRV $=[1 /(a \times$ relação $\mathrm{C} / \mathrm{N}+b \times$ lignina $+c$ x polifenóis $)] \times 100$, em que $a, b$ e $c$ são coeficientes de contribuição relativa da relação $\mathrm{C} / \mathrm{N}(a=0,423)$, dos conteúdos de lignina em porcentagem $(b=0,439)$ e dos polifenóis em porcentagem $(c=0,138)$ para a qualidade do resíduo vegetal.

O estoque de carbono orgânico nas respectivas camadas de solo foi transformado pelo método da massa equivalente de solo (LUCA et al., 2008). Essa metodologia utiliza como referência a massa de solo de um tratamento, a qual é tomada como base para o cálculo do estoque de carbono em todos os demais tratamentos. Neste estudo, foram consideradas como referência as massas de solo das camadas correspondentes da mata nativa, que representa a condição original do solo. Para estimar o carbono orgânico estocado no solo, utilizou-se a fórmu la CS = (PVs x C)/100, proposta por Arevalo et al. (2002), em que CS é o carbono orgânico estocado no solo (em Mg.ha $\left.{ }^{-1}\right)$, PVs é o peso do volume do solo $\left(\mathrm{Mg} \cdot \mathrm{ha}^{-1}\right), \mathrm{C}$ é o teor de carbono em porcentagem analisado em laboratório e 100 é o fator de correção. O PVs foi calculado multiplicando-se a densidade do solo pela espessura do horizonte (em metro) a mostrado e por 10.000 (constante).

Os resultados obtidos foram submetidos à análise de variância, segundo o delineamento inteiramente casualizado, e as médias comparadas pelo teste de Scott-Knott ( $<<0,05)$. Para viabilizar a comparação entre os sistemas, frente ao conjunto de variáveis envolvidas, foi feita análise multivariada, por me io da técnica de análise de conglomerados, pela distância mínima (método de Ward). A medida de similaridade usada para diferenciar as formas de manejo foi a distância euclidiana.

\section{RES ULTADOS E DISCUSS ÃO}

Observou-se que o estoque de serapilheira no período seco deu-se de forma significativa, na ordem mata (MA), sistema agroflorestal (SAF), pasto (PA) e roça de toco (RT), enquanto no período chuvoso a ordem foi SAF, MA, PA e RT (Tabela 1). Os resultados do período seco eram esperados, uma vez que o estoque de serapilheira sobre o solo é dependente da cobertura vegetal predominante no sistema (ALVES et al., 2006; FERREIRA et al., 2007). Como a MA era, notadamente, o sistema com maior biomassa aérea, a deposição de serapilheira sobre esse sistema foi mais expressiva que nas demais formas de uso. Na RT, pelo uso recente do fogo, era esperado baixo acú mu lo de resíduos, conforme o observado.

No período chuvoso, destacou-se o estoque de serapilheira no sistema SAF, com valores até maiores que os encontrados na MA. Esse resultado pode ser decorrente do estágio de sucessão ecológica em que se encontra o sistema (capoeira grossa), que leva a uma renovação intensa de suas folhas em relação à mata (CALDEIRA et al., 2007). A qualidade dos resíduos que compõem a serapilheira também pode ter contribuído com um tempo de residência da serapilheira sobre o solo mais longo nesse sistema em relação à mata (SANCHES et al., 2009), u ma vez que o SAF é u msistema bem menos diversificado. 
Tabela 1. Estoque de serapilheira, carbono na serapilheira, macro e micronutrientes acumulados num Neossolo Quartzarênico em diferentes usos da produção familiar.

Table 1. Litter of stock, carbon in the litter, macro and micronutrients accumulated in Quartzipsamment in different uses of familiar production.

\begin{tabular}{|c|c|c|c|c|c|c|c|c|}
\hline \multirow{2}{*}{$\begin{array}{c}\text { Indicadores } \\
\left(\mathrm{kg}^{2} \mathrm{ha}^{-1}\right)\end{array}$} & \multicolumn{4}{|c|}{ Período seco } & \multicolumn{4}{|c|}{ Período chuvoso } \\
\hline & MA & SAF & $\overline{\mathrm{PA}}$ & RT & MA & SAF & $\mathrm{PA}$ & RT \\
\hline Serapilheira & $11.210 \mathrm{a}$ & $10.670 \mathrm{~b}$ & $8.780 \mathrm{c}$ & $3.830 \mathrm{~d}$ & $6.740 \mathrm{~b}$ & $12.430 \mathrm{a}$ & $4.830 \mathrm{c}$ & $1.260 \mathrm{~d}$ \\
\hline $\mathrm{C}$ & $4.290 \mathrm{a}$ & $4.310 \mathrm{a}$ & $3.320 \mathrm{~b}$ & $1.550 \mathrm{c}$ & $2.270 \mathrm{~b}$ & $4.230 \mathrm{a}$ & $1.750 \mathrm{c}$ & $420 \mathrm{~d}$ \\
\hline $\mathrm{N}$ & $67,28 \mathrm{a}$ & $65,01 \mathrm{a}$ & $55,48 b$ & $30,44 c$ & $81,42 \mathrm{~b}$ & $129,77 \mathrm{a}$ & $38,15 \mathrm{c}$ & $16,87 \mathrm{~d}$ \\
\hline $\mathrm{P}$ & $8,1 \mathrm{~b}$ & $12,66 \mathrm{a}$ & $5,51 \mathrm{c}$ & $2,94 \mathrm{~d}$ & $4,97 \mathrm{~b}$ & $8,47 \mathrm{a}$ & $3,63 \mathrm{c}$ & $1,31 \mathrm{~d}$ \\
\hline K & $70,78 \mathrm{~b}$ & $80,18 \mathrm{a}$ & $51,41 \mathrm{c}$ & $22,77 d$ & $28,48 \mathrm{~b}$ & $54,86 \mathrm{a}$ & $25,98 \mathrm{~b}$ & $7,12 \mathrm{c}$ \\
\hline $\mathrm{Ca}$ & $28,71 \mathrm{a}$ & $22,88 \mathrm{~b}$ & $21,25 b$ & $14,26 \mathrm{c}$ & $45,24 \mathrm{a}$ & $47,43 \mathrm{a}$ & $16,6 \mathrm{~b}$ & $5,88 \mathrm{c}$ \\
\hline $\mathrm{Mg}$ & $11,21 \mathrm{a}$ & $13,67 \mathrm{a}$ & $10,01 \mathrm{a}$ & $4,97 \mathrm{~b}$ & $9,64 \mathrm{~b}$ & $20,8 \mathrm{a}$ & $7,66 \mathrm{~b}$ & $3,1 \mathrm{c}$ \\
\hline$S$ & $2,24 \mathrm{~b}$ & $2,29 \mathrm{~b}$ & $3,02 \mathrm{a}$ & $1,26 \mathrm{c}$ & $9,14 \mathrm{~b}$ & $15,56 \mathrm{a}$ & $6,35 \mathrm{c}$ & $1,75 \mathrm{~d}$ \\
\hline $\mathrm{Cu}$ & $0,25 \mathrm{c}$ & $0,28 \mathrm{~b}$ & $0,31 \mathrm{a}$ & $0,09 \mathrm{~d}$ & $0,11 \mathrm{~b}$ & $0,14 \mathrm{a}$ & $0,04 \mathrm{c}$ & $0,01 \mathrm{~d}$ \\
\hline $\mathrm{Fe}$ & $4,52 \mathrm{a}$ & $4,16 \mathrm{a}$ & $3,13 b$ & $3,04 b$ & $3,98 \mathrm{~b}$ & $8,04 \mathrm{a}$ & $2,85 \mathrm{~b}$ & $0,66 \mathrm{c}$ \\
\hline $\mathrm{Mn}$ & $2,08 \mathrm{a}$ & $1,57 \mathrm{~b}$ & $1,4 b$ & $0,64 \mathrm{c}$ & $1,05 \mathrm{a}$ & $1,39 \mathrm{a}$ & $0,43 \mathrm{~b}$ & $0,12 b$ \\
\hline $\mathrm{Zn}$ & $1,04 \mathrm{a}$ & $0,05 b$ & $0,06 \mathrm{~b}$ & $0,03 \mathrm{c}$ & $0,08 \mathrm{~b}$ & $0,23 \mathrm{a}$ & $0,1 \mathrm{~b}$ & $0,02 \mathrm{c}$ \\
\hline
\end{tabular}

$\mathrm{MA}=$ mata nativa; $\mathrm{SAF}=$ sistema agroflorestal; $\mathrm{PA}=$ pastagem cultivada; $\mathrm{RT}$ = roça de toco. Médias seguidas de mesma letra nas linhas não diferem entre si com o uso do solo para um mesmo período de amostragem, de acordo com o teste de Scott-Knott (p <0,05).

Nos Neossolos Quartzarênicos, elevado aporte de resíduos orgânicos pode representar, entre outras vantagens, proteção aos nutrientes dos efeitos da lixiviação e erosão do solo. Essa é uma condição de explo ração sustentável dessa classe de solos (SALES et al., 2010). Neste estudo, o SAF mostrou elevado potencial.

No que se refere aos nutrientes estocados na serapilheira, no período seco, o SAF foi superior ou semelhante a MA em carbono, nitrogênio, fósforo, potássio, magnésio, en xofre, cobre e ferro, e inferior em cálcio, manganês e zinco, enquanto PA teve valores intermediários e RT os menos expressivos. No período chuvoso, o SAF foi superior aos demais sistemas de uso avaliados em todos os nutrientes e RT o que menos estocou nutrientes em sua serapilheira (Tabela 1).

Esses resultados são decorrentes do elevado aporte de estoque de serapilheira sobre o solo no sistema SAF, pois o acúmulo de nutrientes nesse compartimento é dependente, principalmente, do volume de resíduo aportado pelo sistema (SCHUMACHER, 2004). Ressalte-se o fato de que, no período seco, o SAF acumulou menos resíduos que a MA, mas ainda assim os valores da maioria dos nutrientes em SAF foram iguais ou superiores à mata. A razão para tais resultados reside nas maiores concentrações dos nutrientes nos resíduos do SAF, que é dependente das características das espécies formadoras da serapilheira (FERREIRA et al., 2007), bem co mo da adição de nutrientes via adubação orgânica realizada no SAF.

O carbono foi o constituinte mais expressivo nos resíduos, porque é o principal elemento formador da matéria orgânica. Entre os nutrientes absorvidos pelas plantas do solo, os maiores valores encontrados na serapilheira foram de nitrogênio, potássio, cálcio e magnésio. Isso sugere que os resíduos orgânicos produzidos pelos sistemas são bons recicladores desses nutrientes e alerta para a nutrição com fósforo como um possível limitante na produção desses sistemas, uma vez que, na agricultura familiar da A mazônia, a nutrição com fósforo é dependente da matéria orgânica (VALE JÚNIOR et al., 2011).

No que se refere à qualidade dos resíduos vegetais, o padrão dos resultados da relação C/N e lignina apresenta relação inversa, ou seja, menores valores de C/N implicam maiores de lignina (Figuras 1A e 1B). Nos polifenóis totais, destacaram-se os valores do SAF nos dois períodos de amostragem (Figura 1C), e no índice de qualidade de resíduo vegetal (IQRV) observa-se que MA e PA apresentaram maiores valores no período seco, enquanto no chuvoso o IQRV não difere comos usos do solo avaliados (Figura 1D).

Embora na MA existam muitas espécies lenhosas, o que favorece elevados teores de lignina, a presença de outras espécies ricas em nitrogênio, como as leguminosas, favorece uma menor relação $\mathrm{C} / \mathrm{N}$ quando comparada à obtida na PA. Os elevados valores de polifenóis totais no SAF, em relação aos demais sistemas avaliados nos dois períodos de amostragem, podem estar associados ao domínio das espécies cupuaçu e bacuri nesse sistema de uso.

Os resultados do IQRV, que dá uma ideia comparativa do tempo de residência dos resíduos sobre o solo, sugerem que a serapilheira da MA e do PA, no período seco, são os primeiros a mineralizar. Esses resultados apontam no sentido de que resíduos lenhosos, porém diversificados (como na MA), são equivalentes 
aos monocultivos com pasto no que tange ao tempo de meia-vida sobre o solo. É possível que isso esteja relacionado à especificidade de organismos decompositores necessários para o consumo dos resíduos pouco diversificados da pastagem (SANCHES et al., 2009).

Em geral, os resultados do IQRV encontrados neste estudo são considerados baixos quando comparados aos 4,7 para resíduos de milho e 4,9 para palha de arroz encontrados por Tian et al. (1995), ou aos 8,4 para leguminosas perenes encontrados por Aguiar et al. (2010b). Assim, os resultados deste estudo sugerem que o carbono imobilizado na serapilheira das formas de uso avaliadas, em especial o SAF entre os antropizados, tem um tempo de meia-vida mais longo no solo, o que pode contribuir com a mitigação do efeito estufa.
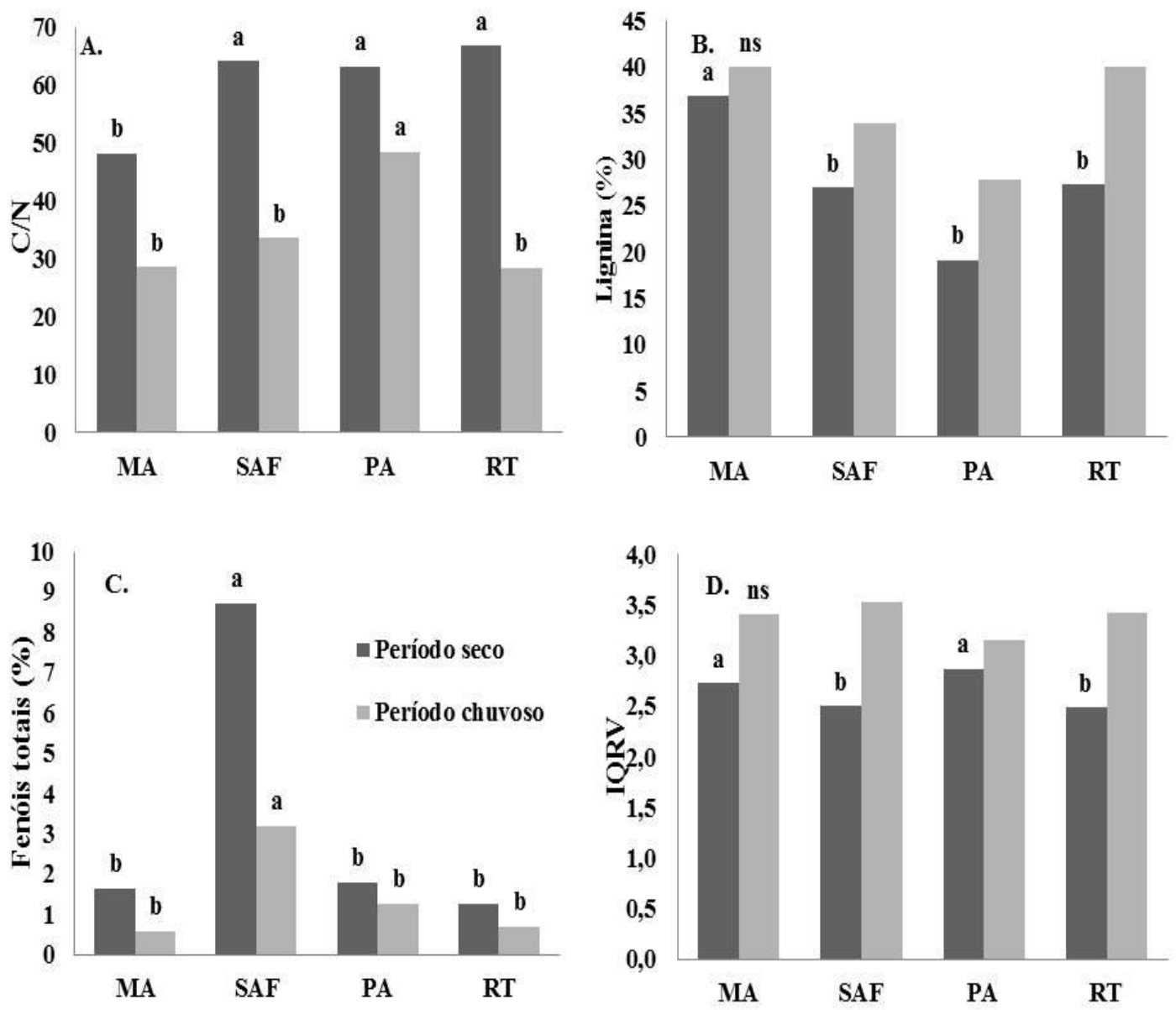

Figura 1. Relação C/N, lignina, fenóis totais e índice de qualidade de resíduos vegetais (IQRV) no período seco e chuvoso num Neossolo Quartzarênico em diferentes usos da agricultura familiar na pré-A mazônia. $\mathrm{MA}=$ mata nativa; $\mathrm{SAF}=$ sistema agroflorestal; $\mathrm{PA}=$ pastagem cultivada; $\mathrm{RT}=$ roça de toco. Médias seguidas de mesma letra não diferem entre si com o uso do solo para um mes mo período de amostragem, de acordo com o teste de Scott-Knott ( $\mathrm{p}<0,05)$.

Figure 1. $\mathrm{C} / \mathrm{N}$ ratio, lignin, total phenols and quality index of plant residues (IQRV) in the dry and rainy seas on in Quartzipsamment in different uses of family farming in the pre-Amazon. MA = native forest; $\mathrm{SAF}=$ agroforestry system; PA = cultivated pasture; RT = stump fields. Means followed by same letter do not differ with the land use for the same sampling period, according to the Scott-Knott test $(\mathrm{p}<0.05)$.

No período seco, RT foi a forma de uso que menos estocou carbono na profundidade $10-20 \mathrm{~cm}$, enquanto o SAF apresentou teores de carbono no solo superiores aos dos demais sistemas na camada $0-5 \mathrm{~cm}$. No período chuvoso não se observaram diferenças significativas entre as formas de uso avaliadas (Tabela 2).

$\mathrm{Na}$ metodologia da massa equivalente de solo, a densidade é usada para equiparar a massa do solo a uma referência, no caso deste estudo, a mata. Assim, o estoque de carbono no solo é dependente, principalmente, do teor de matéria orgânica apresentado nas respectivas camadas. Na camada de $0-5 \mathrm{~cm}$, no período seco, o SAF

FLOREST A, Curitiba, PR, v. 46, n. 1, p. 31 - 38, jan. / mar. 2016.

Freitas, I. C. de et al.

ISSN eletrônico 1982-4688

DOI: $10.5380 / \mathrm{rf.v} 46 \mathrm{il} .42065$ 
apresentou teores de matéria orgânica significativamente superiores, o que resultou em estoque de carbono no solo mais elevado em relação às demais formas de uso. Isso pode estar associado à adubação orgânica recebida anualmente nesse sistema de uso. Em RT, na camada de $10-20 \mathrm{~cm}$, a redução no estoque de carbono pode ser atribuída, em parte, à mineralização de raízes finas após o corte e queima da vegetação natural para o plantio das lavouras. Também é necessário comentar que o aporte de resíduos desse sistema é muito inferior ao dos demais. Esse aporte, possivelmente, não é capaz de sustentar os estoques de carbono no solo, que tendem a cair rapidamente. Outro ponto é o tempo de conversão da mata para RT, que, provavelmente, ainda não se encontra em equilíbrio dinâmico.

Tabela 2. Estoque de carbono calculado pelo método da massa equivalente de solo em dois períodos de amostragem num Neos solo Quartzarênico em diferentes usos da agricultura familiar.

Table 2. Carbon stock calculated by the method of soil equivalent mass in two sampling periods a Quartzipsamment in different uses of family farming.

\begin{tabular}{|c|c|c|c|c|c|c|}
\hline \multirow[b]{2}{*}{ Usos } & \multirow[b]{2}{*}{$\begin{array}{l}\text { Densidade } \\
\left(\mathrm{g} \cdot \mathrm{cm}^{-3}\right)\end{array}$} & \multirow[b]{2}{*}{$\begin{array}{c}\text { Carbono } \\
\text { orgânico }(\%)\end{array}$} & \multicolumn{4}{|c|}{ Massa equivalente de solo } \\
\hline & & & $\begin{array}{l}\text { Camada } \\
(\mathrm{cm})\end{array}$ & \multicolumn{3}{|c|}{$\left(\mathrm{Mg} \cdot \mathrm{ha}^{-1}\right)$} \\
\hline \multicolumn{7}{|c|}{ Período seco } \\
\hline$\overline{\mathrm{MA}}$ & $1,42 \mathrm{~b}$ & $1,42 \mathrm{~b}$ & 0 a 5 & 700 & 9,94 & $\mathrm{~b}$ \\
\hline SAF & $1,23 \mathrm{c}$ & $2,14 \mathrm{a}$ & 0 a 5,7 & 700 & 14,98 & $\mathrm{a}$ \\
\hline PA & $1,51 \mathrm{a}$ & $1,34 \mathrm{c}$ & 0 a 4,6 & 700 & 9,38 & $\mathrm{~b}$ \\
\hline RT & $1,50 \mathrm{a}$ & $0,95 \mathrm{~d}$ & 0 a 4,7 & 700 & 6,65 & $\mathrm{~b}$ \\
\hline$\overline{\mathrm{MA}}$ & $1,45 \mathrm{a}$ & $0,94 \mathrm{~b}$ & 5 a 10 & 725 & 6,81 & $\mathrm{a}$ \\
\hline SAF & $1,37 \mathrm{~b}$ & $1,20 \mathrm{a}$ & 5,7 a 11 & 725 & 8,7 & $\mathrm{a}$ \\
\hline PA & $1,50 \mathrm{a}$ & $1,07 \mathrm{c}$ & 4,6 a 9,4 & 725 & 7,76 & $\mathrm{a}$ \\
\hline RT & $1,46 \mathrm{a}$ & $0,79 \mathrm{c}$ & 4,7 a 9,7 & 725 & 5,73 & $\mathrm{a}$ \\
\hline$\overline{\mathrm{MA}}$ & $1,45 \mathrm{a}$ & $0,67 \mathrm{a}$ & 10 a 20 & 1.450 & 9,72 & $\mathrm{a}$ \\
\hline SAF & $1,37 \mathrm{c}$ & $0,70 \mathrm{a}$ & 11 a 21,6 & 1.450 & 10,15 & $\mathrm{a}$ \\
\hline PA & $1,48 \mathrm{a}$ & $0,80 \mathrm{a}$ & 9,4 a 19,2 & 1.450 & 11,6 & $\mathrm{a}$ \\
\hline RT & $1,40 \mathrm{~b}$ & $0,51 \mathrm{~b}$ & 9,7 a 20,1 & 1.450 & 7,39 & $\mathrm{~b}$ \\
\hline$\overline{\mathrm{MA}}$ & $1,46 \mathrm{a}$ & $0,38 \mathrm{~b}$ & 20 a 40 & 2.920 & 11,1 & $\mathrm{a}$ \\
\hline SAF & $1,37 \mathrm{~b}$ & $0,53 \mathrm{a}$ & 21,6 a 42,9 & 2.920 & 15,48 & $\mathrm{a}$ \\
\hline PA & $1,44 \mathrm{a}$ & $0,39 \mathrm{~b}$ & 19,2 a 39,5 & 2.920 & 11,39 & $\mathrm{a}$ \\
\hline$\underline{\mathrm{RT}}$ & $1,40 \mathrm{~b}$ & $0,26 \mathrm{~b}$ & 20,1 a 41 & 2.920 & 7,59 & $\mathrm{a}$ \\
\hline \multicolumn{7}{|c|}{ Período chuvoso } \\
\hline$\overline{\mathrm{MA}}$ & $1,35 \mathrm{~b}$ & $0,81 \mathrm{a}$ & 0 a 5 & 675 & 5,46 & $\bar{a}$ \\
\hline SAF & $1,22 \mathrm{c}$ & $1,35 \mathrm{a}$ & 0 a 5,5 & 675 & 9,11 & $\mathrm{a}$ \\
\hline PA & $1,51 \mathrm{a}$ & $0,94 \mathrm{a}$ & 0 a 4,5 & 675 & 6,34 & $\mathrm{a}$ \\
\hline RT & $1,38 \mathrm{~b}$ & $1,02 \mathrm{a}$ & 0 a 4,9 & 675 & 6,88 & $\mathrm{a}$ \\
\hline$\overline{\mathrm{MA}}$ & $1,48 \mathrm{a}$ & $1,61 \mathrm{a}$ & 5 a 10 & 740 & 4,51 & $\overline{\mathrm{a}}$ \\
\hline SAF & $1,41 \mathrm{~b}$ & $0,69 \mathrm{~b}$ & 5,5 a 10,7 & 740 & 5,11 & $\mathrm{a}$ \\
\hline PA & $1,50 \mathrm{a}$ & $0,84 \mathrm{~b}$ & 4,5 a 9,4 & 740 & 6,22 & $\mathrm{a}$ \\
\hline RT & $1,52 \mathrm{a}$ & $0,74 \mathrm{~b}$ & 4,9 a 9,8 & 740 & 5,48 & $\mathrm{a}$ \\
\hline$\overline{\mathrm{MA}}$ & $1,51 \mathrm{a}$ & $0,49 \mathrm{a}$ & 10 a 20 & 1.510 & 7,4 & $\overline{\mathrm{a}}$ \\
\hline SAF & $1,46 \mathrm{~b}$ & $0,44 \mathrm{a}$ & 10,7 a 21 & 1.510 & 6,64 & $\mathrm{a}$ \\
\hline PA & $1,52 \mathrm{a}$ & $0,59 \mathrm{a}$ & 9,4 a 19,3 & 1.510 & 8,91 & $\mathrm{a}$ \\
\hline RT & $1,50 \mathrm{a}$ & $0,56 \mathrm{a}$ & 9,8 a 19,9 & 1.510 & 8,46 & $\mathrm{a}$ \\
\hline$\overline{\mathrm{MA}}$ & $1,56 \mathrm{a}$ & $0,35 \mathrm{a}$ & 20 a 40 & 3.120 & 10,92 & $\mathrm{a}$ \\
\hline SAF & $1,39 \mathrm{~b}$ & $0,29 \mathrm{a}$ & 21 a 43,4 & 3.120 & 9,05 & $\mathrm{a}$ \\
\hline PA & $1,52 \mathrm{a}$ & $0,30 \mathrm{a}$ & 19,3 a 39,8 & 3.120 & 9,36 & $\mathrm{a}$ \\
\hline RT & $1,48 \mathrm{a}$ & $0,32 \mathrm{a}$ & 19,9 a 41 & 3.120 & 9,98 & $\mathrm{a}$ \\
\hline
\end{tabular}

$\overline{\mathrm{MA}}=$ mata nativa; SAF = sistema agroflorestal; $\mathrm{PA}=$ pastagem cultivada; $\mathrm{RT}$ = roça de toco. Médias seguidas de mesma letra nas colunas não diferem entre si com o uso do solo para um mesmo período de amostragem, de acordo com o teste de Scott-Knott (p <0,05).

A ausência de diferenças significativas entre as formas de uso nos estoques de carbono no período chuvoso pode ser explicada pela reduzida influência do manejo adotado na matéria orgânica do solo nesse período de amostragem, que é característico dos sistemas de produção que não revolvem o solo (COSTA et al., 2013). Nessas condições de manejo, o carbono estocado no solo tende a manter-se pouco alterado, conforme 
observado por Luca et al. (2008), Rozane et al. (2010) e Froufe et al. (2011). No entanto, em todas as camadas em que as diferenças foram significativas, o SAF estocou mais carbono e RT menos. Esses resultados colocam os sistemas de manejo do solo com SAF e RT em posições opostas no que se refere à adoção de estratégias de manejo voltadas para a imobilização do carbono no solo.

A análise de agrupamento aplicada aos indicadores de qualidade do solo confirma a separação dos sistemas de manejo estudados. Independentemente da época amostrada, o SAF forma grupo com a MA, indicando semelhança, enquanto RT distancia-se da testemunha (MA), sugerindo diferenças significativas (Figura 2). O estoque de serapilheira sobre o solo e os nutrientes contidos nela são, provavelmente, a razão principal para esses resultados. No período chuvoso, RT e PA formam grupo e distanciam-se da MA, indicando serem as formas de uso mais impactadas pelo manejo adotado, o que pode ser confirmado ao se comparare $\mathrm{m}$ os indicadores nas formas de uso individualmente (Tabelas 1 e 2; Figura 1).

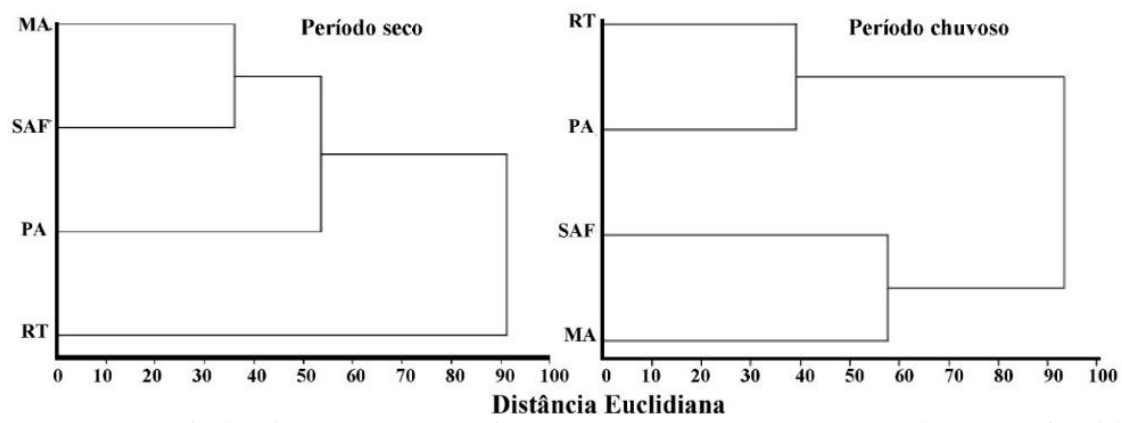

Figura 2. Dendrograma de similaridade entre as formas de uso do solo na agricultura familiar em Neossolo Quartzarênico na pré-Amazônia, agrupadas pelo método de Ward. MA = mata nativa; SAF = sistema agroflorestal; $\mathrm{PA}=$ pastagem cultivada; $\mathrm{RT}=$ roça de toco.

Figure 2. Dendrogram of similarity between the forms of land use in family farming in Quartzipsamment preAmazon, grouped by Ward method. MA = native forest; SAF = agroforestry system; PA = cultivated pasture; RT = stump fields.

Os resultados da análise de agrupamento, usando-se todos os indicadores avaliados, sinaliza que, no período seco, os impactos gerados pelo manejo adotado nos sistemas de uso antropizados deram-se na ordem RT > PA > SAF, enquanto no período chuvoso foi RT $=$ PA > SAF. Esses resultados eram esperados, uma vez que maior intensidade de uso resulta em maiores impactos sobre o solo (SALMI et al., 2009) e apontam o SAF como u ma alternativa de manejo de baixo impacto a longo prazo.

\section{CONCLUSÕES}

- A conversão da floresta em agroecossistemas resultou em alterações na quantidade e qualidade da serapilheira sobre o solo, bem como no carbono estocado no solo.

- O sistema agroflorestal se equiparou à área de mata no estoque de serapilheira, nutrientes na massa seca e carbono estocado no solo, porém seus resíduos apresentaram tempo de meia-vida maior que os da mata.

- O sistema de manejo e uso do solo com roça de toco gerou impactos negativos em todos os indicadores avaliados em relação à mata.

\section{REFERÊNCIAS}

AGUIA R, A. C. F.; BICUDO, S. J.; SObRINHO, J. R. S. C.; MARTINS, A. L. S.; COELHO, K. P.; MOURA, E. G. Nutrient recycling and physical indicators of an alley cropping system in a Sandy loam soil in the préAmazon region of Brazil. Nutrient Cycling in Agroecosytems, A msterdam, v. 86, n. 2, p. 189 - 198, 2010 a.

AGUIA R, A. C. F.; FREITA S, I. C.; CARVAlHO, C. S.; MONROE, P. H. M.; MOURA, E. G. Efficiency of an agrosystem designed for family farming in the pre-A mazon region. Renewable Agriculture and Food Systems, Cambridge, v. 26, n. 1, p. 24 - 30, 2010 b.

ALVES, A. L.; SOUTO, J. S.; HOLANDA, A. C. Aporte e decomposição de serapilheira em área de Caatinga, na Paraíba. Revista de Biologia e Ciência da Terra, Belém, v. 6, n. 2, p. 194 - 203, 2006.

AREVAlo, L. A.; AlegRE, J. C.; VILCAhUAMAN, L. J. M. Metodologia para estimar o estoque de carbono em diferentes sistemas de uso da terra. Colo mbo: Embrapa Florestas, 2002. 41 p. (Documento 73).

FLOREST A, Curitiba, PR, v. 46, n. 1, p. 31 - 38, jan. / mar. 2016.

Freit as, I. C. de et al.

ISSN eletrônico 1982-4688

DOI: $10.5380 /$ rf.v46il 42065 
CALDEIRA, M. V. W.; MARQUES, R.; SOARES, R. V.; BALBINOT, R. Quantificação de serapilheira e de nutrientes em floresta ombrófila mista montana-Paraná. Revista Acadêmica, Curitiba, v. 5, n. 2, p. 101 - 116 , 2007.

COSTA, E. M.; SILVA, H. F.; RIBEIRO, P. R. A. Matéria orgânica do solo e seu papel na manutenção e produtividade dos sistemas agrícolas. Enciclopédia Bi osfera, Goiânia, v. 9, n. 17, p. 1842 - 1860, 2013.

FERREIRA, R. L. C.; LIRA JÚNIOR, M. A.; ROCHA, M. S.; SANTOS, V. S.; LIRA, M. A.; BARRETO, L. P. Deposição e acúmulo de matéria seca e nutrientes em serapilheira em um bosque de sabiá (Mimosa caesalpiniifolia Benth.). Revista Árvore, Viçosa, v. 31, n. 1, p. 7 - 12, 2007.

FROUFE, L. C. M.; RACHWAL, M. F. G.; SEOANE, C. E. S. Potencial de sistemas agroflorestais multiestrata para sequestro de carbono em áreas de ocorrência de Floresta Atlântica. Pesquisa Florestal Brasileira, Colo mbo, v. 31, n. 66, p. 143 - 154, 2011.

LUCA, E. F.; FELLER, C.; CERRI, C. C.; BARTHÈS, B.; CHAPLOT, V.; CAMPOS, D. C. C.; MANECHINI, C. Avaliação de atributos físicos e estoque de carbono e nitrogênio em solos com queima e sem queima de canavial. Revista Brasileira de Ciência do Solo, Viçosa, v. 32, n. 2, p. 789 - 800, 2008.

MATHEUS, M. T. Sequestro de carbono sob a óptica florestal no Brasil. Revista Trópica, Chapadinha, v. 6, n. 1, p. $104-116,2012$.

MORAIS, L. A. C.; MORAIS, V. H. F.; MA GALHÃES, F. E. L.; MOREIRA, A. Emissão de fluxos foliares, floração e ciclagem de nutrientes em clones de copa de Hevea pauciflora. Acta Amazônica, Manaus, v. 42, n. 3, p. $399-404,2012$.

MUTUO, P. K.; CADISCH, G.; ALBRECHT, A.; PALM, C. A.; VERCHOR, M. Potential of agroforestry for carbon sequestration and mitigation of greenhouse gas emissions form soil in the tropics. Nutrient Cycling in Agroecosystems, A msterdam, v. 73, n. 1, p. 43 - 54, 2005.

ROZANE, D. E.; CENTURION, J. F.; ROMUALDO, L. M.; TANIGUCHI, C. A. K.; TRABUCO, M.; ALVES, A. U. Estoque de carbono e estabilidade de agregados de um Latossolo Vermelho distrófico, sob diferentes manejos. Bioscience Journal, Uberlândia, v. 26, n. 1, p. 24 - 32, 2010.

SAlES, L. E. O.; CARNEIRO, M. A. C.; SEVERINO, E. C.; OLIVEIRA, G. C.; FERREIRA, M. M. Qualidade física de Neossolo Quartzarênico submetido a diferentes sistemas de uso agrícola. Ciência e Agrotecnol ogia, Lavras, v. 34, n. 3, p. 667 - 674, 2010.

SALMI, A. P.; SOUZA, A. C.; GUERRA, J. G. M.; RISSO, I. A. M. Teores de nutrientes na biomassa aérea da leguminosa Flemingia macropylla. Revista Brasileira de Agroecologia, Cruz Alta, v. 4, n. 2, p. 873 - 876 , 2009.

SANCHES, L.; VALENTINI, C. M. A.; BIUDES, M. S.; NOGUEIRA, J. S. Dinâmica sazonal da produção e decomposição de serapilheira em floresta tropical de transição. Revista Brasileira de Engenharia Agrícola e Ambiental, Campina Grande, v. 13, n. 2, p. 183- 189, 2009.

SCHUMACHER, M. V. Produção de serapilheira em u ma floresta de Araucaria angustifolia (Bertol.) Kuntze no município de Pinhal Grande/RS. Revista Árvore, Viçosa, v. 28, n. 1, p. 29 - 37, 2004.

SILVA, D. C.; SILVA, M. L. N.; CURI, N.; OLIVEIRA, A. H.; SOUZA, F. S.; MARTINS, S. G.; MACEDO, R. L. G. Atributos do solo em sistemas agroflorestais, cultivo convencional e floresta nativa. Re vista de Estudos Ambientais (online), Blumenau, v. 13, n. 1, p. 77 - 86, 2011.

TIAN, G.; BRUSSAARD, L.; KANG, B. T. An index for assessing the quality of plant residues and evaluation their effects on soil and crop in the sub-humid tropics. Applied Soil Ecology, Amsterdam, v. 13, n. 2, p. 25 - 32, 1995.

VALE JÚNIOR, J. F.; SOUZA, M. I. L; NASCIMENTO, P. R. R.; CRUZ, D. L. S. Solos da Amazônia: etnopedologia e desenvolvimento sustentável. Revista Agro@mbiente On-line, Boa Vista, v. 5, n. 2, p. 158 165,2011

VIEIRA, J. A. G.; TEIXEIRA, M. B.; LOSS, A.; LIMA, E.; ZONTA, E. Produção de serapilheira e ciclagem de nutrientes ao solo pela espécie Eucalyptus urograndis. Revista Brasileira de Agroecologia, Cruz Altas, v. 4, n. 2 , p. $40-43,2009$. 